\title{
MULTISTAGE LOGISTIC REGRESSION MODEL FOR ANALYZING SURVIVAL FROM COLORECTAL CANCER
}

\author{
Yuhaniz Ahmad ${ }^{1 *}$, Zakiyah Zain ${ }^{1,2}$, Nazrina Aziz ${ }^{1,3}$ \\ ${ }^{1}$ School of Quantitative Sciences, Universiti Utara Malaysia, \\ 06010 Sintok, Kedah, Malaysia \\ ${ }^{2}$ Centre of Testing, Measurement and Appraisal (CeTMA), Universiti Utara Malaysia, \\ 06010 UUM Sintok, Kedah, Malaysia \\ ${ }^{3}$ Institute of Strategic Industrial Decision Modelling (ISIDM), Universiti Utara Malaysia, \\ 06010 UUM Sintok, Kedah, Malaysia
}

(Received: July 2018 / Revised: September 2018 / Accepted: December 2018)

\begin{abstract}
Colorectal cancer, which is also known as bowel cancer, colon cancer, or rectal cancer, is the third most common cancer worldwide, and the second most common in Malaysia. In most situations, the tumor/cancer may return even after the tumor removal. The impact of the type of surgery performed for tumor removal on the occurrence of a new tumor and the impact of a patient's state at previous follow-up on the occurrence of a new tumor in the next follow-up are of interest. Thus, the probability of surviving with no tumor recurrence, the probability of surviving but with tumor recurrence, and the probability of not surviving can be obtained. A multistage logistic regression, which was designed for longitudinal data, was used. Data from 161 colorectal cancer patients who experienced tumor removal through surgery and were followed-up for two years were used in this study. The results showed that in the first year after tumor removal surgery, regardless of whether the patients had undergone elective or emergency surgery, the chance of patient survival with no tumor was approximately five times higher than the chance of dying; meanwhile, the chance of dying was approximately three times higher than the chance of developing a new tumor. Similar results have also been obtained for a period of 2 years after the tumor removal surgery.
\end{abstract}

Keywords: Colorectal cancer; Multistage logistic regression; Recurrence; Survival

\section{INTRODUCTION}

This study was motivated by problems with the analysis of life historical data in clinical research. Most existing statistical models are based on concepts according to which a disease instantaneously occurs. Traditional survival analysis deals with analyzing follow-up time: the time elapsed from a starting point until a specific event occurs. Follow-up studies usually stop when the event of interest takes place. However, in most follow-up studies, individuals may experience more than one event at different stages of follow-up. The occurrence rates of these events may be of interest. The traditional survival model, however, is not suitable for analyzing follow-up studies in which more than one event of interest occurred. A multistage model is one of the available methods for analyzing follow-up studies in which more than one event of interest occurs. It can provide a more comprehensive view of a disease process, allowing the estimation of probabilities while making use of incomplete information efficiently and handling

\footnotetext{
*Corresponding author's email: yuhaniz@uum.edu.my, Tel.+604-9286311, Fax.+604-9286309 Permalink/DOI: https://doi.org/10.14716/ijtech.v9i8.2764
} 
loss to follow-up problems appropriately. Examples of diseases from which patients may experience more than one event include all kinds of cancers, myocardial infarction, heart disease, cyst development, and diabetes.

Cancer is an uncontrolled growth of abnormal cells, also known as cancer cells, or malignant tumors anywhere in a body. Cancer cells can break away from their original form, travel through the blood and lymph systems, and invade other organs (Reboux, 2018). To stop the uncontrolled growth cycle of cancer cells, certain treatments must be considered. Surgery, chemotherapy, and radiotherapy are among the options available for the treatment of patients with cancer.

Colorectal cancer, which is also known as bowel cancer, colon cancer, or rectal cancer, is the third most common cancer worldwide. In Malaysia, colorectal cancer is the second most common cancer in males and the third most common cancer in females (Veettil et al., 2017). The incidence and mortality of colorectal cancer are higher in males compared to females. Meanwhile, in terms of ethnicity, those of Chinese ethnicity have the highest incidence of colorectal cancer (Hassan et al., 2016), followed by those of Malay and Indian ethnicities. In Malaysia, most patients with colorectal cancer are diagnosed at a very late stage (Goh et al., 2005; Rashid et al., 2009). This could be due to a lack of awareness of the symptoms and signs of colorectal cancer as well as the increasing incidence of colorectal cancers in Malaysia. Delay in looking for treatment or screening for colorectal cancer detection before any symptoms or signs occur may adversely impact recovery and survival. A national program for colorectal cancer screening should be implemented, especially in low-income communities (Tze et al., 2016)

Even though there are several choices of treatment for colorectal cancer patients, surgery is the most common treatment for tumors. Tumor surgery involves the removal of part of the healthy intestine and the nearby lymph nodes of patients. Medical surgery for tumor removal is suitable not only for younger patients but also for the elderly, as delayed mortality and an early survival rate can be expected among elderly patients (Hobler, 1986). In most situations, the tumor/cancer may recur after treatment, and it may affect the rectum, colon, or any other part of the body even though the surgery has been performed on the patients. Therefore, even after successful surgery, some patients may require chemotherapy to reduce the risk of the tumor returning. In other words, follow-up treatment is always a must for colorectal cancer patients. However, the question of how fast the new tumor can develop once it has been removed remains unanswered. This study examined the effect of the types of surgery that have been performed on patients, and the effect of patients' conditions in the previous follow-up on the chance of new tumor recurrence in the subsequent follow-up.

\subsection{Objectives of the Study}

At every one-year follow-up time (also referred to as stage k), colorectal patients undergo a checkup to determine whether the tumor has returned. If it has, another surgery is performed. Generally, the impact of the type of surgery performed on a patient at time $t_{k-1}$ on the occurrence of a new tumor at time $t_{k}$, and the impact of a patient's state at time $t_{k}$ on the occurrence of a new tumor at $t_{k+1}$, are of interest. Thus, this study aimed to: (1) Model the effect of different types of surgery and the state of colorectal cancer patients in the previous follow-up on the probability of tumor recurrence; and (2) Obtain the probability of surviving with no tumor recurrence, the probability of surviving but with tumor recurrence, and the probability of not surviving. 


\section{METHODOLOGY}

In multistage analysis, the definitions of events are always the same at any follow-up time, and the occurrences of events at previous follow-up times are examined in terms of covariates. The multistage model has been used widely in analyzing cancer development and has been found to be very successful in describing many features of cancer development (Armitage \& Doll, 1954; Moolgavkar, 1978; Holford et al., 1994; Pompei \& Wilson, 2001; Ritter et al., 2003). However, the early version of the multistage model was based on duration or time of occurrence $\left(\mathrm{t}_{\mathrm{i}}\right)$ of an event, and thus it is not suitable for events measured in a categorical manner, such as exist or not exist.

Logistic or probit regression models have the ability to deal with events that are measured in a categorical manner. Both logistic and probit regression models can be used in the exact same situations, and the discrepancy in the overall results of the model are typically extremely slight to non-existent. A logistic regression uses a logit link function, while a probit regression uses an inverse normal link function. The choice between the two methods usually comes down to interpretation and communication. The logit links provide a slightly intuitive way to interpret the effects of a factor, while interpreting the probit link is more difficult.

In traditional logistic regression, the duration or time of occurrence $\left(\mathrm{t}_{\mathrm{i}}\right)$ of an event is ignored in the model. A logistic regression model is an appropriate procedure for describing the effect of several numbers of categorical or continuous explanatory variables on the probability of occurrences of a dichotomous outcome variable (Peng et al., 2002). Logistic regression has been applied in many different areas, such as in medicine (Yusuff et al., 2012; Zangmo \& Tiensuwan, 2018), engineering (Barus et al., 2016), and social sciences (Ramosacaj et al., 2015). However, most follow-up studies have more than one type of event; thus, the traditional logistic model is no longer suitable for use. Therefore, a multistage logistic model (Islam et al., 2004) is preferred.

A multistage logistic model is based on a conditional approach and uses the occurrences of previous events efficiently. It is an extension of logistic regression for the covariate-dependent Markov model (Muenz \& Rubinstein, 1985) that can be implemented not to only first order but also to a higher order covariate dependence. It can be employed to solve problems in modeling transitions, reverse transitions, and repeated transitions. One of the advantages of the multistage logistic model is that the definition of events is not necessarily the same but can also change with respect to the stage, and the occurrences of events are examined at follow-up times within a stage. A multistage model often provides more insight into a study and can give more precise survival estimates. In a multistage model, the transition between any two adjoining states of an event is modeled by either a Markov or a semi-Markov process. A semi-Markov model can be considered as a generalization of the Markov model.

\subsection{Preparation of Multistage Logistic Model in Colorectal Cancer Patients' Data}

In general, each colorectal cancer patient who has undergone the first surgery for tumor removal is considered as being in state $y=1$ at year 0 (stage 0 ). Each patient may develop a new tumor (also known as tumor recurrence) in the next one-year follow-up (also known as stage 1), may not develop a new tumor, or may die. If the patient experiences tumor recurrence in stage 1, a new surgery is performed to remove the new tumor. In the next one-year follow-up (stage 2), colorectal cancer patients once again undergo a checkup to determine whether another new tumor has developed. The state transition from stage 0 to stage 1 , and from stage 1 to stage 2 , is shown in Figure 1. For example, patients can remain in the same state 1 (survive with no tumor recurrence) throughout the study period (later denoted by 1-1-1) or can move from state 1 to state 2 (tumor recurrence) in year 1 and finally state 0 (died) in year 2 (later denoted by 1-2$0)$. Details of state transitions are shown in Figure 1. 


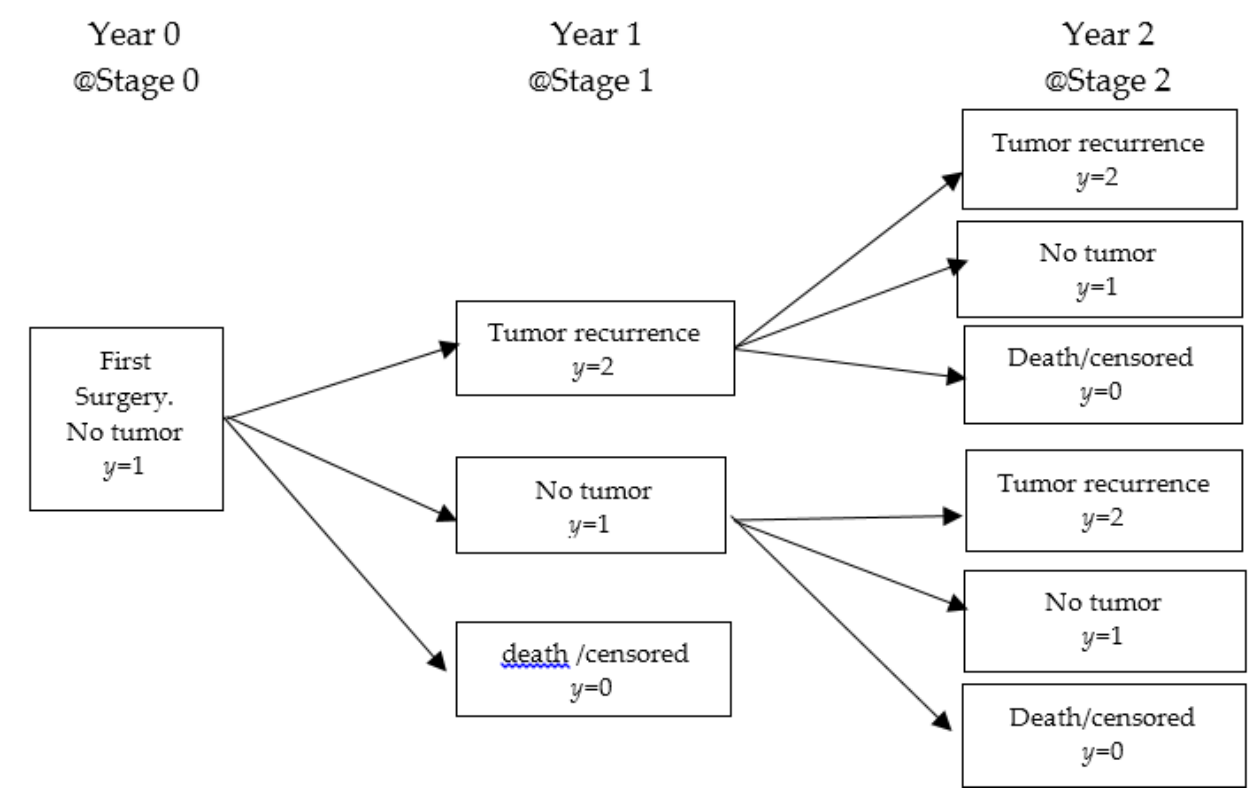

Figure 1 State transition at different stages of observation

To model the transition state $\mathrm{Y}_{\mathrm{mk}}$ of patients, from stage (k-1) to stage (k), and from stage (k-1) to stage $(k)$ to stage $(k+1)$, we can define $Y_{m k}=i$, where $Y_{m k}=2$ if a tumor recurrence event occurred at the observed stage $\mathrm{k}$ for the $\mathrm{m}^{\text {th }}$ patient $\mathrm{m}=1,2,3, \ldots, \mathrm{n}_{\mathrm{k}} ; \mathrm{Y}_{\mathrm{mk}}=1$ if a patient survived with no tumor recurrence; and $\mathrm{Y}_{\mathrm{mk}}=0$ if a patient did not survive or was loss to follow-up. Generally, the logistic regression model for an event at $\mathrm{k}^{\text {th }}$ stage can be written as:

$$
p\left(Y_{m k}=i / Z_{m k}\right)=\frac{e^{\beta_{k} z_{m k}}}{1+e^{\beta_{k}^{\prime} z_{m k}}}
$$

where $\beta_{k}^{\prime}$ for $k=1,2$ is the vector of parameters corresponding to the vector of covariate $Z_{k}$. Generally, the vector of covariates is denoted as:

- For $k=1$ (stage 1$), Z_{1}=\left[z_{1}, z_{2}, \ldots, z_{p}\right]$;

- For $k=2$ (stage 2), $Z_{2}=\left[z_{1}, z_{2}, \ldots, z_{p}, z_{p+1}\right]$, where $z_{p+1}=2,1,0$ for any events that take place during stage 1 . Considering the first order Markov model, only one previous event is considered as a covariate in the model.

The logit of the probability of the existence of tumor recurrence at stage 1 can be defined as a linear function of covariates:

$$
g_{1}(Z)=\ln \left[\frac{p\left(Y_{m 1}=2 / Z_{m 1}\right)}{p\left(Y_{m 1}=0 / Z_{m 1}\right)}\right]=\beta_{1}{ }^{\prime} Z_{m 1}
$$

Meanwhile, the logit of the probability of surviving with no tumor recurrence at stage 1 can be defined as a linear function of covariates:

$$
g_{1}(Z)=\ln \left[\frac{p\left(Y_{m 1}=1 / Z_{m 1}\right)}{p\left(Y_{m 1}=0 / Z_{m 1}\right)}\right]=\beta_{1}{ }^{\prime} Z_{m 1}
$$

which gives the probability of each event at different stages for a given covariate vector as:

$$
p\left(Y_{m k}=i / Z_{m k}\right)=\frac{e^{\beta_{k}{ }^{\prime} z_{m k}}}{1+e^{\beta_{k} z_{m k}}}=\pi_{m k}\left(Z_{m k}\right)
$$

As given by Islam et al. (2004), the likelihood contribution of the $k$ th stage can be written as: 


$$
L_{k}(\beta)=\prod_{m=1}^{n_{k}}\left[\pi_{m k}\left(Z_{m k}\right)\right]^{Y_{m k}}\left[1-\pi_{m k}\left(Z_{m k}\right)\right]^{1-Y_{m k}}
$$

Thus, the overall likelihood function and the log likelihood function is given by:

$$
L(\beta)=\prod_{k=1}^{2} \prod_{m=1}^{n_{k}}\left[\pi_{m k}\left(Z_{m k}\right)\right]^{Y_{m k}}\left[1-\pi_{m k}\left(Z_{m k}\right)\right]^{1-Y_{m k}}
$$

The estimation of the $p$ th parameter of the transition at the $k$ th stage is computed by:

$$
\frac{\delta \ln L(\beta)}{\delta \beta_{p i u}^{k}}=0, p=1 ., 2, \ldots, p+k-1 ; i, u=0,1 ; k=1,2
$$

\section{RESULTS AND DISCUSSION}

This study focused on the effect of surgery treatment and the state of patients in the previous follow-up on the probability of tumor recurrence among colorectal cancer patients. Table 1 gives the summary of the data based on gender, ethnic groups, age at diagnosis, and stage at

\begin{tabular}{|c|c|c|c|}
\hline \multicolumn{2}{|c|}{ Profile } & \multirow{2}{*}{$\frac{\text { Frequency }}{84}$} & \multirow{2}{*}{$\frac{\text { Percentage }}{52 \%}$} \\
\hline & Male & & \\
\hline Gender & Female & 77 & $48 \%$ \\
\hline \multirow{4}{*}{ Ethnicity } & Malay & 59 & $37 \%$ \\
\hline & Chinese & 96 & $60 \%$ \\
\hline & Indian & 5 & $3 \%$ \\
\hline & Others & 1 & $1 \%$ \\
\hline \multirow{4}{*}{ Age at Diagnosis } & $15-29$ & 2 & $1 \%$ \\
\hline & $30-49$ & 21 & $13 \%$ \\
\hline & $50-64$ & 75 & $47 \%$ \\
\hline & 65 and over & 63 & $39 \%$ \\
\hline \multirow{4}{*}{ Stage at Diagnosis } & 1 & 2 & $2 \%$ \\
\hline & 2 & 54 & $45 \%$ \\
\hline & 3 & 41 & $34 \%$ \\
\hline & 4 & 24 & $20 \%$ \\
\hline
\end{tabular}
diagnosis.

Table 1 Summarized profile data of 161 colorectal cancer patients

As displayed in Table $1,52 \%$ of the patients were males and more than one-half $(60 \%)$ were Chinese. Since this study was based on a real dataset, the number of patients by ethnicity was not controlled. In Malaysia, as reported in 2016, the Chinese ethnicity has the highest incidence of colorectal cancer, followed by the Malay and Indian ethnicities. This finding is consistent with those reported elsewhere (Hassan et al., 2016). Thus, our sample is representative of the Malaysian population. Furthermore, the effect of ethnicities was not of interest in this study, as it focused only on the effect of past surgery for tumor removal and the type of surgery undergone by patients on the probability of tumor recurrence. Most patients (79\%) were diagnosed with colorectal cancer when they were already at stage 2 or 3 , and almost $90 \%$ of the patients were diagnosed at age 50 or above. These results are consistent with those reported by other researchers (Hassan et al., 2016; Veettil et al., 2017; Magaji et al., 2017). More than 65\% of the 161 patients were still alive during the study period, while $32 \%$ had died or had been lost to follow-up.

The status of surgery for each patient, namely emergency $\left(Z_{m l}=0\right)$ versus elective $\left(Z_{m l}=1\right)$, and tumor recurrence $\left(Z_{m 2}=0,1\right)$ were considered the two potential factors in this study. Table 2 gives the number of patients with recurrent tumor at each stage. Most surgeries performed were chosen electively $(63.4 \%)$, where decisions were made by patients with their physicians after 
considering the best options available for the stage and location of the cancer, as well as the risks and benefits associated with age and other factors; meanwhile, the remaining 36.6\% underwent emergency surgery (See Table 2).

Table 2 Percentage of patients with recurrent tumor

\begin{tabular}{cccc}
\hline $\begin{array}{r}\text { Types of } \\
\text { Surgery }\end{array}$ & \multicolumn{2}{c}{ Patients with tumor recurrence } & $\begin{array}{c}\text { Total patients } \\
\text { undergoing surgery }\end{array}$ \\
\cline { 2 - 3 } Emergency & Stage 1 & Stage 2 & 59 \\
& 3 & 6 & $(36.6 \%)$ \\
\hline \multirow{2}{*}{ Elective } & $(5.1 \%)$ & $(10.2 \%)$ & 102 \\
& $(5.9 \%)$ & $(8.8 \%)$ & $(63.4 \%)$ \\
\hline Total & 9 & 15 & 161 \\
\hline
\end{tabular}

Furthermore, it can be concluded that most of the patients survived with no tumor recurrence after the first and second year from surgery, as only nine out of $59(15.3 \%)$ and 15 out of 102 $(14.7 \%)$ patients who underwent emergency and elective surgeries, respectively, experienced tumor recurrence in the first two years after their first surgery. The percentage of patients who experienced tumor recurrence at stage 2 was higher than at stage 1 regardless of the type of surgery, either emergency or elective.

The transition from one stage to another stage was of interest in this study. The number of patients who moved to a state of tumor recurrence, state of no tumor recurrence, and censored/death state in stage $k$ will be denoted as $y_{m k}$, where $y_{m k}=2,1,0$, respectively. Out of the 161 colorectal cancer patients considered in this study, 28\% died (transition type 1-0 and 1-1-0) before the new tumor developed, while 3\% died (transition type 1-2-0) in the second year after tumor recurrence in the first year, meaning that a total of $31 \%$ of patients died within two years of the first tumor removal surgery. The number of events together with the percentage of events are shown in Table 3.

Table 3 Number of events by types of transition

\begin{tabular}{ccc}
\hline $\begin{array}{c}\text { Types of transition } \\
\text { Stage 0- Stage 1- Stage 2 }\end{array}$ & $\begin{array}{c}\text { Number of } \\
\text { events }\end{array}$ & $\begin{array}{c}\text { Percentage of } \\
\text { events }\end{array}$ \\
\hline $1-0$ & 28 & $17 \%$ \\
$1-1-0$ & 18 & $11 \%$ \\
$1-1-1$ & 91 & $57 \%$ \\
$1-1-2$ & 15 & $9 \%$ \\
$1-2-0$ & 5 & $3 \%$ \\
$1-2-1$ & 4 & $2 \%$ \\
\hline
\end{tabular}

Within two years after surgery for tumor removal, more than one-half ( $57 \%$ with transition type 1-1-1) of the patients survived with no tumor recurrence. On the other hand, only $14 \%$ (transition type 1-1-2, 1-2-0,1-2-1) of the patients developed a new tumor within the first two years of observation. Out of the $14 \%$ of patients who experienced tumor recurrence within the first two years of the first tumor removal surgery, 9\% (transition type 1-1-2) had tumor recurrence in their second year, while the other 5\% (transition type 1-2-0 and 1-2-1) experienced it in their first year. 


\subsection{Coefficients of Multistage Logistic Regression Model}

The multistage logistic regression model fit the data very well for both stage 1 and stage 2 . As stated in Table 4, the logistic regression model for both stage 1 and stage 2 was significant, with a $p$-value $=0.000$. In stage 1 , both types of treatment received during the removal surgery had a significant effect on patient survival with no tumor recurrence. However, the difference in the effect between the two treatments was very small. The effects of both treatments on tumor recurrence when compared to surviving with no tumor were totally different. The probability of each event occurring at different stages for a given covariate vector can be found by substituting the significant coefficient value into the model (3). The probability of a patient developing a new tumor was low, while the probability of death was higher. On the other hand, the probability of surviving with no tumor was higher than the probability of death in the first year after tumor removal surgery. More specifically, in the first year after tumor removal surgery, regardless of whether patients underwent elective or emergency surgery, the probability of surviving with no tumor was approximately five times higher than the probability of dying, while the probability of dying was approximately three times higher than the probability of developing a new tumor.

Table 4 Estimates of parameter from multistage logistic model

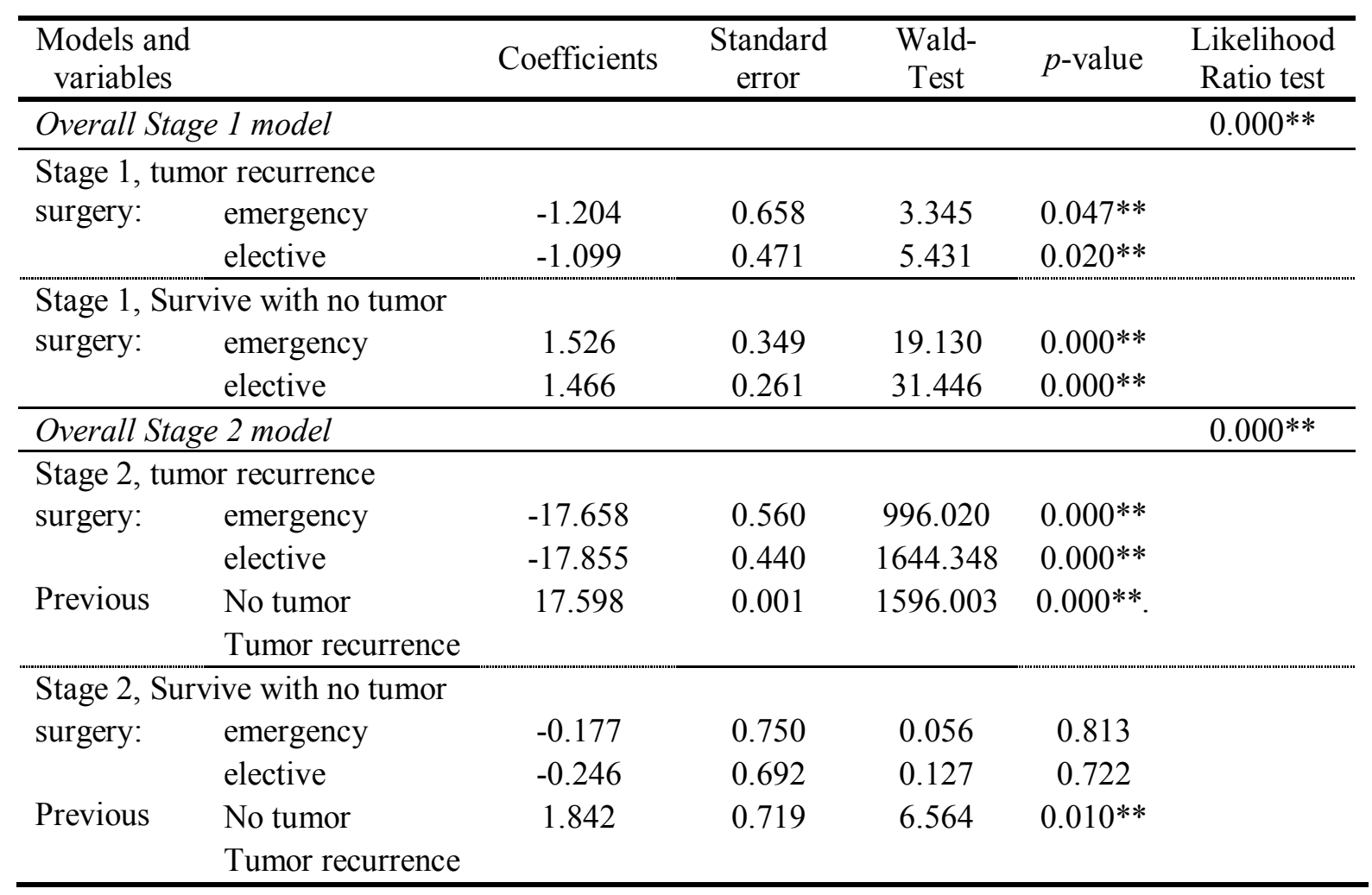

** Significant at $5 \%$

In stage 2, both types of surgery had a significant effect on reducing the chance of tumor recurrence. Considering that the patients had undergone an emergency surgery, this finding shows that when the patients had no tumor recurrence in the first year after the first surgery, their chance of developing a new tumor was slightly lower than the chance of dying. In the second model of stage 2, both types of surgery had no significant effect on increasing or reducing the chance of survival with no tumor in stage 2 . Thus, the chance of surviving with no tumor was also higher than the chance of dying if the patients had no tumor recurrence in the first year after the first surgery. Like the conclusion made based on the model in stage 1, we can 
also conclude that in the second year after tumor removal surgery, the chance of a patient surviving with no tumor was approximately six times higher than the chance of dying, while the chance of dying was approximately 1.1 times higher than the chance of developing a new tumor.

In both stages, once the tumor has been removed, the chances of surviving with no tumor was higher than the chances of dying. This result is comparable to that in other Asian nations (Abu Hassan et al., 2016) but lower than that found in more developed countries (Magaji et al., 2017). Apart from the factors considered in this study, there are many other factors that might affect the survival rate of cancer patients. Approximately 15 to $30 \%$ of colorectal cases may be due to genetic factors (Dove-Edwin \& Thomas, 2001). However, habits such as smoking, alcohol use, and low fruit and vegetable intake are the leading risk factors for death from cancer (Danaei et al., 2005; Ghee, 2014). Staying as healthy as possible and at a healthy weight, being physically active, eating a healthy diet with an emphasis on plant foods, and avoiding alcohol should be a priority for staying healthy (Dove-Edwin \& Thomas, 2001). These steps may lower the risk of tumor recurrence as well as other health problems.

\section{CONCLUSION}

The profile summary of the 161 hospital cases is consistent with that of the reported Malaysian data. Mainly, (i) Chinese had the highest incidence compared to other ethnicities, (ii) more males than females were diagnosed with cancer, (iii) most cases were diagnosed at the age of 50 or above. Out of the 161 colorectal cancer patients considered in this study, $28 \%$ died before the new tumor developed, while 5\% died in the second year after tumor recurrence in their first year. Within two years after surgery for tumor removal, about one-half $(57 \%)$ of the patients survived with no tumor recurrence.

In the main year of development or after one year of tumor removal, regardless of whether an elective or emergency medical procedure was performed, the probability of survival with no tumor was around five times higher than the probability of dying, while the probability of dying was roughly three times higher than the probability of tumor recurrence. A similar conclusion was made in view of the model in stage 1; accordingly, we can likewise infer that in the second year after the tumor removal medical procedure, the probability of survival with no tumor was around six times higher than the probability of dying, while the probability of dying was roughly 1.1 times higher than the probability of developing another tumor. This suggests that undergoing a medical procedure for tumor removal is superior to leaving the tumor in place, as surgery can boost the probability of survival.

As life expectancy continues to increase in Malaysia, as in the rest of the world, it is believed that the rate of colorectal cancer cases will continue to rise. If Malaysians continue to ignore the increasing incidence of colorectal cancer, there will be a lower chance of early detection and survival. Educating the public, especially high-risk groups for cancer, is necessary to increase awareness of colorectal cancer. A national program for colorectal cancer screening should be implemented, especially in low-income communities, to increase early detection and improve the survival rate of colorectal cancer patients. As the popular proverb goes, prevention is better than a cure. Various agencies working hand in hand with early screening for low-income communities could hopefully lower the colorectal cancer incidence rate in this demographic.

Finally, this study demonstrates that a multistage logistic model is suitable for studying patients' progress in longitudinal studies, especially when follow-ups are made in the same time interval. The model has been widely used to make analysis easier, as it also allows different definitions of states in different stages. 


\section{ACKNOWLEDGEMENT}

The authors would like to thank Universiti Utara Malaysia (RAGS S/O 12686), UKM Medical Centre (HUKM), and the National Registration Department of Malaysia (JPN) for their support in this research.

\section{REFERENCES}

Abu Hassan, M.R., Ismail, I., Mohd Suan, M.A., Ahmad, F., Wan Khazim, W.K., Othman, Z., Mat Said, R., Tan, W.L., Mohammed, S.R., Soelar, S.A., Nik Mustapha, N.R., 2016. Incidence and Mortality Rates of Colorectal Cancer in Malaysia. Epidemiology and Health, Volume 38, pp. 1-5

Armitage, P., Doll, R., 1954. The Age Distribution of Cancer and a Multi-stage Theory of Carcinogenesis. British Journal of Cancer, Volume 8(1), pp. 1-12

Barus, L.S., Flores, H.M., Hadiwardoyo, S.P., Batoz, J.L., 2016. Intercity Mode Choice Modelling: Considering the Intracity Transport Systems with Application to the JakartaBandung Corridor. International Journal of Technology, Volume 7(4), pp. 581-591

Danaei, G., Vander Hoorn, S., Lopez, A.D., Murray, C. J., Ezzati, M., 2005. Causes of Cancer in the World: Comparative Risk Assessment of Nine Behavioural and Environmental Risk Factors. The Lancet, Volume 366(9499), pp. 1784-1793

Dove-Edwin, I., Thomas, H.J., 2001. Review Article: The Prevention of Colorectal Cancer. Alimentary Pharmacology and Therapeutics, Volume 15(3), pp. 323-336

Ghee, L.K., 2014. A Review of Colorectal Cancer Research in Malaysia. Medical Journal of Malaysia, Volume 69, pp. 23-32

Goh, K.L., Quek, K.F., Yeo, G.T., Hilmi, I.N., Lee, C.K., Hasnida, N., Aznan, M., Kwan, K.L., Ong, K.T., 2005. Colorectal Cancer in Asians: A Demographic and Anatomic Survey in Malaysian Patients Undergoing Colonoscopy. Alimentary Pharmacology and Therapeutics, Volume 22(9), pp. 859-864

Hassan, M.R., Suan, M.A., Soelar, S.A., Mohammed, N.S., Ismail, I., Ahmad, F., 2016. Survival Analysis and Prognostic Factors for Colorectal Cancer Patients in Malaysia. Asian Pacific Journal of Cancer Prevention, Volume 17(7), pp. 3575-3581

Hobler, K.E., 1986. Colon Surgery for Cancer in the very Elderly; Cost and 3-year Survival. Annals Surgery, Volume 203(2), pp. 129-131

Holford, T.R., Zhang, Z., McKay, L.A., 1994. Estimating Age, Period and Cohort Effects using the Multistage Model for Cancer. Statistics in Medicine, Volume 13(1), pp. 23-41

Islam, M.A., Chowdhury, R.I., Chakraborty, N., Bari, W., 2004. A Multistage Model for Maternal Morbidity during Antenatal, Delivery and Postpartum Periods. Statistics in Medicine, Volume 23(1), pp. 137-158

Magaji, B.A., Moy, F.M., Roslani, A.C., Law, C.W., 2017. Survival Rates and Predictors of Survival among Colorectal Cancer Patients in a Malaysian Tertiary Hospital. BMC Cancer, Volume 17(1), pp. 339-346

Moolgavkar, S.H., 1978. The Multistage Theory of Carcinogenesis and the Age Distribution of Cancer in Man. The Journal of the National Cancer Institute, Volume 61(1), pp. 49-52

Muenz, L.R., Rubinstein, L.V., 1985. Markov Models for Covariate Dependence of Binary Sequences. Biometrics, Volume 41(1), pp. 91-101

Peng, C.Y.J., Lee, K.L., Ingersoll, G.M., 2002. An Introduction to Logistic Regression Analysis and Reporting. The Journal of Educational Research, Volume 96(1), pp. 3-14

Pompei, F., Wilson, R., 2001. Age Distribution of Cancer: The Incidence Turnover at Old Age. Human and Ecological Risk Assessment, Volume 7(6), pp. 1619-1650 
Ramosacaj, M., Hasani, V., Dumi, A., 2015. Application of Logistic Regression in the Study of Students' Performance Level. Journal of Educational and Social Research, Volume 5(3), pp. 239-244

Rashid, M.R., Aziz, A.F., Ahmad, S., Shah, S.A., Sagap, I., 2009. Colorectal Cancer Patients in a Tertiary Referral Centre in Malaysia: A Five Year Follow-up Review. Asian Pacific Journal Cancer Preview, Volume 10(6), pp. 1163-1166

Reboux, G., 2018. Cancer. World Health Organisation. Available Online at https://www.who.int/en/news-room/fact-sheets/detail/cancer, Accessed on October 30, 2018

Ritter, G., Wilson, R., Pompei, F., Burmistrov, D., 2003. The Multistage Model of Cancer Development: Some Implications. Toxicology and Industrial Health, Volume 19(7-10), pp. $125-145$

Tze, C.N., Fitzgerald, H., Qureshi, A., Tan, H.J., Low, M.L., 2016. Pioneering Annual Colorectal Cancer Screening and Treatment Targeting Low Income Communities in Malaysia (2010-2015). Asian Pacific Journal of Cancer Prevention, Volume 17(7), pp. 3179-3183

Veettil, S.K., Lim, K.G., Chaiyakunapruk, N., Ching, S.M., Abu Hassan, M.R., 2017. Colorectal Cancer in Malaysia: Its Burden and Implications for a Multiethnic Country. Asian Journal of Surgery, Volume 40(6), pp. 481-489

Yusuff, H., Mohamad, N., Ngah, U.K., Yahaya, A.S., 2012. Breast Cancer Analysis using Logistic Regression. International Journal of Recent Research and Applied Studies (IJRRAS), Volume 10(1), pp. 14-22

Zangmo, C., Tiensuwan, M., 2018. Application of Logistic Regression Models to Cancer Patients: A Case Study of Data from Jigme Dorji Wangchuck National Referral Hospital (JDWNRH) in Bhutan. Journal of Physics: Conference Series, Volume 1039, pp. 1-10 